\title{
Flipping a Coin: Theory and Evidence ${ }^{1}$
}

\author{
Nadja Dwenger \\ Max Planck Institute for Tax Law and Public Finance Munich \\ and \\ Dorothea Kübler \\ Social Science Research Centre Berlin (WZB) \\ \& Technical University Berlin \\ and \\ Georg Weizsäcker $\left.\right|^{2}$ \\ Humboldt Universität Berlin \\ \& German Institute of Economic Research (DIW Berlin)
}

\footnotetext{
${ }^{1}$ A previous version of this paper was entitled "Preference for Randomization: Empirical and Experimental Evidence" (Working Paper of the MPI for Tax Law and Public Finance No.14, 2012). We thank the ZVS - particularly Bernhard Scheer, Harald Canzler and Uwe Kuhnen - for providing us with the university admission data set and for additional information. We are grateful for helpful comments by Eddie Dekel, Frank Heinemann, and audiences at Birmingham, Essex, Innsbruck, MPI Jena, SUNY, TU Berlin, Toulouse, UCL, Zurich and HEC Paris. Financial support by the DFG (CRC 649 "Economic Risk") and the ERC (Starting Grant 263412) is gratefully acknowledged.

${ }^{2}$ Contact: weizsaecker@hu-berlin.de
} 


\title{
Flipping a Coin: Theory and Evidence
}

\begin{abstract}
We investigate the possibility that a decision-maker prefers to avoid making a decision and instead delegates it to an external device, e.g., a coin flip. In a series of experiments the participants often choose lotteries between allocations, which contradicts most theories of choice such as expected utility but is consistent with a theory of responsibility aversion that implies a preference for randomness. A large data set on university applications in Germany shows a choice pattern that is also consistent with this theory and entails substantial allocative consequences.
\end{abstract}

Keywords: Preference for randomization, menu-dependent preference, individual decision making, university choice, matching

JEL Classification: D03; D01 


\section{Introduction}

In situations where decision-making is hard, a possible procedural preference arises: the decision-maker may wish for the decision to be taken away from herself. Her cognitive or emotional cost of deciding may outweigh the benefits that arise from making the optimal choice. For example, the decision-maker may prefer not to make a choice without having sufficient time and energy to think it through. Or, she may not feel entitled to make it. Or, she may anticipate a possible disappointment about her choice that can arise after a subsequent resolution of uncertainty. Waiving some or all of the decision right may seem desirable in such circumstances even though it typically increases the chance of a suboptimal outcome.

The difficulty of such preferences is that they are non-consequentialist and are therefore excluded by most models of choice such as expected utility. In particular, flipping a coin between different choice options contradicts expected utility theory except if the decisionmaker is exactly indifferent between these options. Yet people regularly do flip coins or revert to other random decision aids $3^{3}$ More general than expected utility theory, two closely related basic axioms of choice - stochastic dominance and betweenness - postulate that whenever the decision-maker has a strict preference for one of the options, she makes the choice herself rather than delegate it to randomness $\mathbb{4}^{4}$

This paper discusses preferences that allow for coin flipping. It then presents several data

\footnotetext{
${ }^{3}$ For example, preferences for randomization can be inferred from a number of successful products carrying a random feature: surprise menus at restaurants, last minute holiday booking desks at airports, movie sneak previews that do not advertise the movie titles, surprise-me features of Internet services, and home delivery of produce bins with variable content. For a historical account of randomization in the political arena see Buchstein (2009).

${ }^{4}$ Both axioms require that preference is preserved in probabilistic mixtures. In our context, their main difference lies in the domain of preference; stochastic dominance relates to sure outcomes whereas betweenness relates to lotteries. The stochastic dominance property is, in the version of Borah (2010): if $a \succ b$ and $c \succsim d$ hold for outcomes $a, b, c, d$, then a probabilistic mixture between the two preferred outcomes is preferred to a mixture of the two less preferred outcomes, $(\lambda a,(1-\lambda) c) \succ(\lambda b,(1-\lambda) d)$, where $\lambda \in(0,1]$ is the common probability of receiving the first object. Betweenness requires that if $\mathcal{L} \succ \mathcal{L}^{\prime}$ holds for two lotteries $\mathcal{L}, \mathcal{L}^{\prime}$, then the preference value of $\left(\lambda \mathcal{L},(1-\lambda) \mathcal{L}^{\prime}\right)$ lies between that of the two lotteries.
} 
sets, experimental and field-empirical, that are consistent with a preference for randomization and inconsistent with expected utility. The largest data set concerns an important choice in people's lives: university applications. When applying to the German clearinghouse for university admission in one of the centrally administered fields of study, the (fairly complicated) quota rules require each applicant to submit multiple rank-order lists of universities knowing that each of the lists could be relevant for determining her university seat allocation. Our empirical observation is that between the multiple submitted rank-order lists, university applicants often contradict themselves. Thereby, they effectively delegate the outcome to an uncertain process that is suboptimal under standard assumptions. If an applicant had always reported the same rank-order list instead of producing a preference reversal between her submitted lists, she would have increased the probability of ending up at a more desirable university. Our cleanest (and most conservative) calculation, which excludes rational reasons for choice reversals (see Section 4.3.3), finds that $14 \%$ of applicants exhibit such behavior. Among these, we estimate that at least $20 \%$ are assigned to a university that they prefer less than a university to which they would have been assigned in the absence of a preference reversal.

Our theoretical discussion follows the psychological literatures on decision avoidance, regret, and responsibility (see e.g. Beattie et al. (1994) and Anderson (2003)), emphasizing the psychic costs of decision-making. If a random device is available and if the process of using the random device does not violate other principles, decision-makers have been shown to use it in hypothetical scenarios (Keren and Teigen 2010). More generally, the psychological literature argues that decision-makers often prefer to avoid a decision even if this comes at the cost of a (probabilistic) material consequence. We model the desire to avoid decisions by defining "responsibility averse" decision-makers.$^{5}$ A responsibility averter behaves like an

\footnotetext{
${ }^{5}$ See Leonhardt et al. (2011) who define responsibility aversion as a wish to minimize one's causal role in determining an outcome. In an experiment with hypothetical choices over outcomes for others they observe that lotteries are chosen and that participants state that they feel less responsible for an outcome that resulted from a lottery.
} 
expected-utility maximizer but she also has the desire to choose an action that is unlikely to be ex-post suboptimal. In a state of the world where her choice is suboptimal, she would feel responsible for the bad outcome. Anticipating this possible regret, she prefers a choice that involves randomness because she can blame the random device ex post. Our assumption is, thus, very similar to regret theory (Loomes and Sudgen 1982) with the important addition that the use of randomization relieves the decision-maker from possible regret. As discussed e.g. by Zeelenberg and Pieters (2007), this tendency to employ randomization can be viewed as stemming from a particular omission bias, where decision-makers associate active decisions with more regret than inactive choices such as a coin flip 6 By symmetry, we also allow for the opposite property of "responsibility loving": a responsibility lover prefers a daring choice that has a larger likelihood of being suboptimal, as she anticipates the possible elation from having made a good choice that could have turned out to be suboptimal: 7

Interestingly, economists pay much less attention to the possibility of intentional use of random devices than psychologists. Diecidue et al. (2004) argue that this is due to precisely the property of stochastic dominance violations: a theory that comes with a feature that is normatively so unappealing as stochastic dominance violations is easily dismissed. Diecidue et al. provide a different interpretation of coin flipping - namely utility of gambling - and prove that stochastic dominance violations are a core feature of such preferences 8

Not making a decision may of course be optimal even under standard preferences. Especially informational reasons often justify the use of one of three avoidance strategies: delay, default, or delegation. It may be important for the decision-maker to remain flexible if poten-

\footnotetext{
${ }^{6}$ See also Kahneman and Miller (1986) and Gilovich and Medvec (1995) for discussions of inaction and regret.

${ }^{7}$ Yet, responsibility lovers cannot be detected in our data sets without making further assumptions on preferences because a responsibility lover, just like an expected-utility maximizer, never flips a coin.

${ }^{8}$ Our theoretical discussion in Section 2 differs from the model of Diecidue et al. (2004) and related papers in that we assume that all outcomes are uncertain. We assume that there prevails some uncertainty regarding the outcomes stemming from the available decisions. Our theory therefore yields a possible violation of betweenness, not stochastic dominance (see Footnote 4). However, leaving our theory aside, one can interpret the observed preference for randomness as a stochastic dominance violation if one views a given allocation (university matching and experimental voucher) as a sure outcome.
} 
tial news can arrive in the future (delay). Or, there may exist an exogenous agent or decision mechanism that uses better information, making it optimal to leave the choice to them (default or delegation). But these reasons are unlikely to apply in the case of university choice as the applicants know that their rank-order lists cannot be changed after the arrival of new information and that the mechanism selecting the lists does not utilize much choice-relevant information. Yet another possible rationalization of the preference reversals in university choice - one that we cannot rule out - are irrational beliefs of the decision-makers. The allocation mechanism of the central clearinghouse is highly complicated and depends on the submitted preferences of thousands of applicants 9 Allowing for the possibility of non-rational beliefs on behalf of the applicants, a large set of choice patterns can be optimal.

We therefore conduct a set of simple choice experiments, most of them run in large undergraduate lectures, that are suitable to test the implications of our theory in an incentivized setting. The experimental rules and procedures are straightforward and leave little room for ambiguity or false beliefs. A caveat is that in low-stake classroom experiments, the psychic costs of decision-making are likely small. But this does not rule out that the cost of making the optimal choice is larger than its benefit. Indeed we deliberately use decision problems where the typical participant is close to indifferent - she chooses between vouchers from different shops that have similar monetary values. We observe that substantial proportions of participants (between $15 \%$ and $53 \%$ ) choose a random outcome, violating standard theory. Our theoretical discussion correctly predicts several systematic patterns in the data. The alternative hypothesis that participants are simply indifferent between the available choices trivially allows for any choice but does not specifically predict the observed patterns. Moreover, the hypothesis that undirected random utility shocks drive behavior is rejected by the

\footnotetext{
${ }^{9}$ The mechanism is analyzed in Braun et al. (2010), Braun et al. (2012) and Westkamp (2013), pointing to a particular strategic feature of the mechanism: the fact that the mechanism involves a sequence of procedures affects the truth-telling incentives at the early stages because the applicants have to consider the effect of moving to the next procedure. In this paper we use these and other results from the literature on matching algorithms to rule out the possibility that strategic effects may lead to preference reversals that look like a preference for randomization.
} 
data. 10 We therefore conclude that the observed choice of randomized outcomes appears to be deliberate.

Several of our experiments and the university application process share the feature that the decision-makers choose between the same options repeatedly. In these experiments, the participants choose twice and a random draw determines which of the two choices is payoff relevant. The design thereby allows the participants to put one option ahead of another one in their first choice and vice versa in their second choice. We observe that sizable numbers of participants (15-40\%, in different treatments) show such reversals. The simplest version of this is the binary-choice format of Experiment I (Section 3.1), where two options are available on each of two choice lists and $28 \%$ of participants indicate a preference reversal between their two choices. In a control group that makes the choice between the options only once, we observe that the average choice leans more heavily in favor of one option.

But the experimental evidence is not limited to choice reversals. A separate treatment (Experiment II, Section 3.2 shows that when participants are offered an explicit option to randomize between the options, they choose it in $53 \%$ of cases. The frequency of choosing such coin flipping is higher than in a control treatment where the same options are made available through a repeated-choice format like the one in Experiment I. Finally, Experiment III (Section 3.3 confirms that reversals can also appear in situations where people have to rank more than just two options, as in the context of university admissions.

Two strands of the experimental economics literature have previously generated evidence on non-material preferences regarding randomization. One set of evidence considers procedural concerns in the context of fairness. People do care about procedures and not just the outcomes, especially when multiple people are involved. For example, they have preferences over different allocation mechanisms even if they themselves do not participate in the mech-

\footnotetext{
${ }^{10}$ An important literature analyzes whether previously found biases in decision experiments may be generated by models of random choice. See e.g. Berg et al. (2010) for a study on preference reversals.
} 
anism (Kahneman et al. 1986). Famously, the procedure becomes more important than the outcome in the example of "Machina's mom" (Machina 1989). She decides to flip a coin in order to decide which of her two children gets an indivisible goody although she has a preference for one of the children getting it rather than the other child. Flipping the coin is more preferable to her (or rescues her from bearing the consequences of showing her preference order). Experimental results confirm that people may prefer ex ante fair lotteries if an ex post fair outcome is unavailable (see Bolton et al. (2005) and Krawczyk and Le Lec (2010)) ${ }^{11}$

The second set of related experimental studies concerns cases where decision-makers put unnecessarily high probability on undesirable outcomes. Camerer and Ho (1994) report on experiments between multiple lotteries where the betweenness axiom is violated. In experiments that are most closely related to our study and that were conducted independently, Agranov and Ortoleva (2013) demonstrate that subjects who face the same binary-lottery choice task several times in a row often switch between their choices if one lottery is not clearly better than the other one. They also observe that a significant fraction of subjects is willing to pay for a coin flip between the two lotteries. Other studies show that experimental participants sometimes value a lottery less than its worst possible realization (Gneezy et al. (2006), Sonsino (2008), Andreoni and Sprenger (2011)) ${ }^{12}$ While some of this evidence goes in the opposite direction of our findings, we note that our proposed effect of indecision and responsibility aversion may be small in these previous studies as their participants do not have the choice between two simple options and a probabilistic mixture between the two. Another related set of previous experiments concerns a false diversification motive (see Chen and Corter (2006) and Rubinstein (2002)), where participants choose mixtures in ways that violate expected

\footnotetext{
${ }^{11}$ To account for such evidence, Borah (2010) develops a model where a decision-maker's utility is a weighted sum of her expected utility from the lottery outcome as well as a procedural component. Sen (1997) proposes accommodating concerns for the procedure in menu-dependent models where preferences over outcomes can depend on the menu or set of outcomes available.

${ }^{12}$ For instance, in Gneezy et al. (2006) participants in one group choose between a sure 100 shekels in cash and a lottery that offers a fifty-fifty lottery between a 200 shekel and a 400 shekel gift certificate. In another treatment, participants choose between a sure 100 shekels in cash and a 200 shekel gift certificate. The choice percentage of the lottery is smaller than the choice percentage of the lottery's worst outcome.
} 
utility. In contrast to these studies, our participants can get at most one reward, erasing the possibility of a diversification value, and the understanding of our experiments is cognitively very straightforward. Thus, a false belief in diversification would have to be quite false in our experiments and we therefore propose interpreting the data as participants knowing what they do and wanting to do it 13

The remainder of the paper proceeds as follows. In Section 2, the assumption of responsibility responsiveness is formulated in a context of menu-dependent choice. Section 3 describes our series of experiments together with their results. Section 4 introduces the empirical context of university choice in Germany. To identify the subset of data that is suitable for our purposes, the section discusses and applies the relevant literature on matching mechanisms. Finally, it shows the calculations that yield our main empirical result. Section 5 concludes.

\section{Theory: Assumptions on responsibility preference}

The following describes a domain of preferences and behavioral assumptions that are consistent with intentional coin flips ${ }^{14}$ Let $S=\left\{s_{1}, \ldots, s_{N}\right\}$ be a finite set of states of the world and let $\mathcal{A}=\left\{a_{1}, \ldots\right\}$ be a menu of available actions, which is a subset of the "grand set" of actions, $\Gamma=\bigcup \mathcal{A}$. Each action is a mapping from the set of states to the set of material payoffs $\Pi$, such that the material payoff $\pi(a, s) \in \Pi$ of choosing action $a \in \mathcal{A}$ depends on the realized state $s \in S$; the decision-maker makes her pick from $\mathcal{A}$ before she can learn the realized s. For example, the material consequences of applying to a particular university depend on unknown contingencies that are collected in $S$. Likewise, the ex-post value of owning a given voucher (our experimental reward) depends on the unknown state $s \in S$. Below, we will

\footnotetext{
${ }^{13}$ Rubinstein (2002) also documents a false diversification in a context where only one prize can be earned. He finds an irrational mixture of choices and ascribes the effect to a cognitive failure of grasping the multi-stage randomness in his experiment (wrongly applying the intuition of diversification).

${ }^{14}$ We do not provide a complete axiomatization of behavior with a representing function. Instead, we state basic assumptions directly on the preference relation and argue that this statement suffices to guide our choice of statistical hypotheses in the subsequent sections.
} 
consider menus containing three elements: two "normal" actions $a_{1}$ and $a_{2}$ (e.g. applications to university 1 and university 2 ) as well as a lottery $\mathcal{L}_{1,2}$ that allows the decision-maker to randomize between $a_{1}$ and $a_{2}$. We will ask whether it is possible that she strictly prefers $\mathcal{L}_{1,2}$ over both $a_{1}$ and $a_{2}$.

In order to make the lottery $\mathcal{L}_{1,2}$ available for an arbitrary state space $S$, we augment $S$ by combining it with the set $\{H, T\}$ : we construct $\widetilde{S}=S \times\{H, T\}$, denote the elements of $\widetilde{S}$ by $(s, H)$ and $(s, T)$, respectively, and interpret this augmentation as saying that there exists an external device that selects $\mathrm{H}$ (eads) and implements action $a_{1}$ or it selects $\mathrm{T}($ ails $)$ and implements $a_{2}$. Such coin flipping between $a_{1}$ and $a_{2}$ is available as a choice option if the menu contains an action $\mathcal{L}_{1,2}$ with payoff $\pi\left(\mathcal{L}_{1,2},(s, H)\right)=\pi\left(a_{1},(s, H)\right)=\pi\left(a_{1},(s, T)\right)$ and $\pi\left(\mathcal{L}_{1,2},(s, T)\right)=\pi\left(a_{2},(s, H)\right)=\pi\left(a_{2},(s, T)\right)$, for all $s \in S{ }^{15}$

We enable a desire for randomization by assuming that the decision-maker's preference is menu dependent. She has, for each possible menu $\mathcal{A}$, a separate preference relation $\succsim_{\mathcal{A}}$. The non-standard feature of menu dependence is restricted to one particular characteristic: like in regret theory, we assume that the decision-maker reacts to the possibility that her action is ex-post suboptimal. We measure this possibility of suboptimality by the following set.

Definition 1. The responsibility set of action $a \in \mathcal{A}$ is

$$
m(a, \mathcal{A})=\left\{s \in S: \exists a^{\prime} \in \mathcal{A} \text { with } \pi\left(a^{\prime},(s, j)\right)>\pi(a,(s, j)) \text { for all } j \in\{H, T\}\right\} .
$$

In words, the responsibility set is the set of states $s$ in which $a$ is strictly suboptimal. Conditional on having chosen $a$, these states can lead to regret if they materialize or-as the opposite of regret - they may lead to contentment if they fail to materialize. Notice that

\footnotetext{
${ }^{15}$ Notice that the external device may not follow a set of objectively known probabilities. One can think of it as another person to whom the decision-maker delegates her choice. Delegation of a decision right to another person is, for instance, studied in Bartling and Fischbacher (2010), who identify delegation as an effective device for avoiding punishment for unpopular decisions.
} 
if $a=\mathcal{L}_{1,2}$ is a lottery between actions $a_{1}$ and $a_{2}$, then its responsibility set is relatively small: for all states in which $a_{1}$ or $a_{2}$ are weakly payoff optimal, the lottery cannot be strictly suboptimal for both $(s, H)$ and $(s, T)$. This corresponds to the observation that if the decisionmaker chooses $\mathcal{L}_{1,2}$, she can blame the lottery for the outcome. That is, $m\left(\mathcal{L}_{1,2}, \mathcal{A}\right) \subseteq m\left(a_{i}, \mathcal{A}\right)$ for all $i \in\{1,2\}$.

Our main definition below allows the decision-maker to react to $m(a, \mathcal{A})$. She follows a preference relation $\succsim_{\mathcal{A}}$ that is derived from a (standard, menu-independent) "reference preference" $\succsim$. We assume throughout that the preference relation $\succsim$ satisfies the expected utility axioms for choice under uncertainty ${ }^{16}$ The only non-standard assumption is that $\succsim_{\mathcal{A}}$ may differ from $\succsim$ if the sets $m(a, \mathcal{A})$ and $m\left(a^{\prime}, \mathcal{A}\right)$ are nested:

Definition 2. (i) The decision-maker is responsibility loving if $a \succsim a^{\prime}$ implies $a \succsim_{\mathcal{A}} a^{\prime}$, for all $\mathcal{A} \in \Gamma$ and all $a, a^{\prime} \in \mathcal{A}$ with $m\left(a^{\prime}, \mathcal{A}\right) \subseteq m(a, \mathcal{A})$.

(ii) The decision-maker is responsibility averse if $a^{\prime} \succsim$ a implies $a^{\prime} \succsim_{\mathcal{A}}$ a, for all $\mathcal{A} \in \Gamma$ and all $a, a^{\prime} \in \mathcal{A}$ with $m\left(a^{\prime}, \mathcal{A}\right) \subseteq m(a, \mathcal{A})$.

The definition of responsibility aversion captures the idea that an action $a$ is less preferred if it is strictly suboptimal in more states of the world than an alternative action $a^{\prime}$. The formulation is weak in the sense that the preference between $a$ and $a^{\prime}$ may still be identical between the two relations $\succsim$ and $\succsim_{\mathcal{A}}$, but it is also possible that a larger responsibility set of $a$ induces a preference shift to the disadvantage of $a$. Conversely, the assumption of the agent being responsibility loving describes a decision-maker who prefers action $a$ weakly more if it is suboptimal under more states of the world. This captures a preference for making a daring choice. The case of menu-independent preferences (expected utility) is nested in our setup by

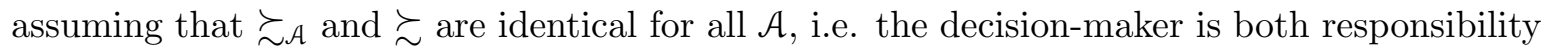
loving and responsibility averse.

\footnotetext{
${ }^{16}$ See e.g. the textbook presentation in Wakker (2010), Chapter 4.
} 
The following proposition shows that being responsibility loving precludes a preference for randomization, in the sense that no lottery in $\Gamma$ is strictly preferred to all of its components, and that responsibility aversion enables a preference for randomization. All proofs are in the appendix.

Proposition 1. Let $\mathcal{A}=\left\{a_{1}, a_{2}, \mathcal{L}_{1,2}\right\}$ be the menu of available actions, and assume that neither $a_{1}$ nor $a_{2}$ are weakly optimal in all states $s \in S$. (i) A decision-maker who is responsibility loving cannot strictly prefer $\mathcal{L}_{1,2}$ over both $a_{1}$ and $a_{2}$. (ii) There exist responsibility averse decision-makers who strictly prefer $\mathcal{L}_{1,2}$ over both $a_{1}$ and $a_{2}$.

Notice that the definitions of responsibility loving and responsibility averse use only weak (preference) inequalities. Proposition 1 therefore gives only qualitative results. But this suffices to indicate directions for the statistical tests that we apply in the subsequent sections, to be formulated in highlighted hypotheses below.

A further notable property of our analysis is that we do not insert a "biased" assumption regarding the preference for or against responsibility. It is conceivable that equal proportions of decision-makers are, respectively, responsibility averters and responsibility lovers. Nevertheless, Proposition 1 shows that we can observe a systematic deviation from the standard prediction: the responsibility lovers will be indistinguishable from responsibility neutral agents, in terms of the observed preference for randomization — but the responsibility averters will tend to seek randomness.

The following proposition shows that in addition to Proposition 1, responsibility averters have a particular tendency between two available lotteries: if they choose to randomize, they will pick a lottery that favors their most-preferred non-random item.

Proposition 2. Let $\mathcal{A}=\left\{a_{1}, a_{2}, \mathcal{L}_{1,2}, \mathcal{L}_{2,1}\right\}$ be the available menu, where $\mathcal{L}_{i, j}$ is a lottery that assigns probability $p>\frac{1}{2}$ to action $a_{i}$ and probability $1-p$ to action $a_{j}$. Assume that all preferences are strict on $\Gamma$ and assume w.l.o.g. that $a_{1} \succ a_{2}$. Then a responsibility averse 
decision-maker prefers $\mathcal{L}_{1,2}$ over $\mathcal{L}_{2,1}$.

The proposition illustrates that a systematic direction of preference reversals is predicted for responsibility averters. This pattern can be subject to experimental tests.

\section{Experiments}

This section presents our three experimental designs and results. The design of Experiment III is closest to the university application context of the subsequent section, whereas Experiments I and II are stripped-down versions of Experiment III but can also stand alone as tests for a possible effect of responsibility aversion. We run all experiments outside of the laboratory as we can investigate one-shot individual decision tasks more efficiently in other settings. We employ classroom experiments as well as a newspaper experiment in combination with an online survey. While the participants in the classroom experiment are students, a more diverse population is reached with the newspaper experiment. All experiments are incentivized.

The recruitment of participants differs between the three experiments. The classroom experiments (Experiment I, Experiment II, and parts of Experiment III) were conducted in introductory undergraduate lectures in microeconomics and macroeconomics at Technical University Berlin and Free University Berlin. The newspaper experiment (the remaining part of Experiment III) was advertised in the "WZB-Mitteilungen", a quarterly journal of the social science research institute WZB. The WZB-Mitteilungen is read by researchers, policy makers, and the interested public ${ }^{17}$

\footnotetext{
${ }^{17} \mathrm{~A}$ translation of the experimental instructions of Experiment II is in the appendix and the (very similar) instructions of the other experiments are available upon request.
} 


\subsection{Experiment I: Deliberate choice reversals}

\subsubsection{Design}

In Treatment 1 of Experiment I, 69 participants face a choice between two prizes. Prize $a_{1}$ is a combined package of a 14 Euro Starbucks voucher together with a 10 Euro Amazon voucher. The other prize, $a_{2}$, is a 19 Euro Starbucks voucher 18 Participants have to choose twice between the two prizes, but only one choice task is relevant ex post: participants receive the prize that they report in the first choice task with probability 0.6 and the prize that they report in the second choice task with probability 0.4 .

Proposition 1 applies to this context by the observation that two lotteries between $a_{1}$ and $a_{2}$ are available: the participants can choose to report one prize in the first choice task and the other prize in the second choice task. No responsibility lover (or expected-utility maximizer) with strict preferences will induce randomness, i.e., all responsibility lovers will choose the same object twice. Among the responsibility averters, some may choose a lottery. Proposition 2 shows that under responsibility aversion, the less-preferred object tends to be chosen in the second choice task (realized with lower probability), not the first.

In a control treatment, Treatment 2, we let a separate set of 68 participants choose only once between the two prizes $a_{1}$ and $a_{2}$. For comparison with the second choice task in Treatment 1 , the payout rule is that participants receive their chosen prize with probability 0.4. Treatment 2 generates a comparison data set, allowing us to observe the participant population's preference between the two prizes. Under standard preferences, the same choice frequencies would arise on average for the second choice task in Treatment 1 and the choice task in Treatment 2. The same holds true in a model where in each of the two tasks, the

\footnotetext{
${ }^{18}$ The choice of vouchers as prizes has several motivations. (i) The description and delivery of vouchers are straightforward both in the classroom experiments and in the newspaper experiment. (ii) Monetary values of vouchers are flexible. (iii) Vouchers carry an inherent uncertainty with respect to their ex-post consumption outcomes, making them suitable for our analysis.
} 
utility of each choice is perturbed by a random utility shock.

Since no randomization between $a_{1}$ and $a_{2}$ is possible in Treatment 2, we take choices in Treatment 2 to reflect the reference preference $\succsim$. Letting $\bar{f}_{1, c}\left(a_{i}\right)$ denote the frequency with which participants choose prize $a_{i}$ in choice task $c \in\{1,2\}$ in Treatment $1, \bar{f}_{1}\left(a_{i}, a_{j}\right)$ the frequency of two different choices $\left(a_{i}, a_{j}\right)$ in the two choice tasks of Treatment 1 (made by the same participant), and $\bar{f}_{2}\left(a_{i}\right)$ the frequency of $a_{i}$ in Treatment 2 , we collect the relevant results in a behavioral hypothesis:

HYPOTHESIS 1: As per Proposition 1, responsibility averters may intentionally submit two different choices in the two tasks of Treatment $1: \bar{f}_{1}\left(a_{1}, a_{2}\right)+\bar{f}_{1}\left(a_{2}, a_{1}\right)>0$. As per Proposition 2, they will pick their less preferred object in the second choice task of Treatment 1, not the first. Moreover, they will choose their less preferred object more often in the second choice task of Treatment 1 than in Treatment 2. Thus, if $\bar{f}_{2}\left(a_{1}\right)>\frac{1}{2}$ (indicating a preference for $a_{1}$ according to $\left.\succsim\right)$, then $\bar{f}_{1,2}\left(a_{1}\right)<\bar{f}_{1,1}\left(a_{1}\right)$ and $\bar{f}_{1,2}\left(a_{1}\right)<\bar{f}_{2}\left(a_{1}\right)$. Conversely, if $\bar{f}_{2}\left(a_{2}\right)>\frac{1}{2}$, then $\bar{f}_{1,2}\left(a_{2}\right)<\bar{f}_{1,1}\left(a_{2}\right)$ and $\bar{f}_{1,2}\left(a_{2}\right)<\bar{f}_{2}\left(a_{2}\right)$.

Both treatments are run in parallel in the same classroom, by randomly distributing different sets of instructions to different students. We paid out a prize to every tenth participating student: at the end of the experiment, we randomly determined the participants who would receive a prize and played out the random draws for the two treatments 19

\subsubsection{Results}

In Treatment 2, we observe that $81 \%$ of participants choose $a_{1}$. Thus, Hypothesis 1 predicts for Treatment 1 that $\bar{f}_{1,2}\left(a_{1}\right)<\bar{f}_{2}\left(a_{1}\right)=0.81$ and $\bar{f}_{1,2}\left(a_{1}\right)<\bar{f}_{1,1}\left(a_{1}\right)$.

Figure 1 shows the different choice combinations made by the participants in Treatment

\footnotetext{
${ }^{19}$ It may be that the random payment procedure relieves the participants from their responsibility preferences. This would make it less likely that the effects predicted by our theory appear.
} 
Figure 1: Choice combinations made in Treatment 1

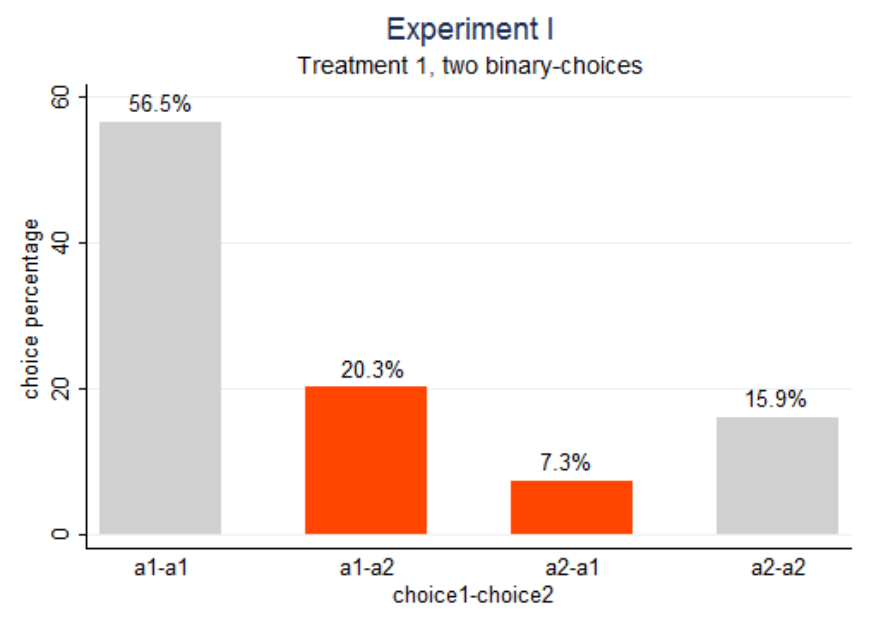

1. Hypothesis 1 is fully supported in these data. The first observation is that a significant proportion of participants reverse their choices: $28 \%$ of participants either choose $a_{1}$ in the first task and $a_{2}$ in the second task or the reverse. While such choice reversals could be trivially explained by indifference, we observe that the systematic predictions of Hypothesis 1 are also confirmed: First, the choice of $a_{1}$ in choice task 2 of Treatment 1 is significantly less frequent than in Treatment $2,0.64=\bar{f}_{1,2}\left(a_{1}\right)<\bar{f}_{2}\left(a_{1}\right)=0.81(p=0.02$, Fisher's exact test). Second, in Treatment 1 the share of participants choosing $a_{1}$ in the first choice task is larger than in the second choice task ( $p=0.03$, one-sided test on the equality of matched pairs of observations).

RESULT 1: 28\% of participants submit two different choices in the two tasks of Treatment 1. In line with Hypothesis 1, participants tend to pick their most preferred choice in the first choice task and their less preferred choice in the second choice task.

As described in Section 3.1.1., a random perturbation of utilities would predict a different pattern of choices. Under this assumption, choices in the second task of Treatment 1 would be equally distributed as choices in Treatment 2, which is not observed. The presence of the first choice task significantly affects the choice probability in the second choice task. 


\subsection{Experiment II: explicit preference for randomness}

\subsubsection{Design}

Experiment II is a framing experiment. 166 participants have the choice between two prizes, where prize $a_{1}$ is a 19 Euro Amazon voucher and prize $a_{2}$ is a 19 Euro Starbucks voucher. We conduct two treatments varying the choice format. In the binary-choice format of Treatment 3, 74 participants choose twice between the two prizes just as in Treatment 1 of Experiment I. Once again, only one choice task counts in the sense that the choice reported in the first choice task becomes relevant with probability 0.6 and the choice reported in the second choice task with probability 0.4 . Treatment 4 is economically equivalent to Treatment 3 but it has a four-way-choice format where 94 participants face the explicit choice between the following four options: $: 20$

Option 1: You receive $a_{1}$ with probability 0.6 and $a_{2}$ with probability 0.4 .

Option 2: You receive $a_{1}$ with probability 1 .

Option 3: You receive $a_{2}$ with probability 0.6 and $a_{1}$ with probability 0.4 .

Option 4: You receive $a_{2}$ with probability 1 .

As the randomization opportunity becomes more salient in Treatment 4, we conjecture that Treatment 4 evokes the participants' responsibility attitudes more strongly than the twoway choice frame of Treatment 3 . If this is the case, the frequency of dominance violations should be even larger in Treatment 4, as formulated in the following hypothesis, where $\bar{f}_{t}(k)$ refers to the frequency with which Option $k \in\{1,2,3,4\}$ is chosen in Treatment $t \in\{3,4\}$.

HYPOTHESIS 2: By Proposition 1, responsibility averters may submit two different choices in Treatment 3. In Treatment 4, even more participants may choose to randomize,

\footnotetext{
${ }^{20}$ In the experimental instructions, we phrase the probabilities in percentages and provide a description of the probabilities in terms of the frequency that a certain event occurs when 100 draws from an urn are made.
} 
i.e. $\bar{f}_{4}(1)+\bar{f}_{4}(3)>\bar{f}_{3}(1)+\bar{f}_{3}(3)>0$.

Both treatments of Experiment II are run in the same classroom and one out of 20 participants is paid out for real at the end of the experiment.

\subsubsection{Results}

As is shown in Figure 2, $28 \%$ of the participants randomize in Treatment 3 by choosing both A and B exactly once in the two tasks. This compares to $53 \%$ of the participants in Treatment 4 who choose the explicit option to randomize between the two different alternative prizes, i.e., Option 1 or Option 3. In the four-way-choice treatment the share of randomizing individuals is significantly larger compared to the binary-choice treatment $(p<0.01$, one-sided Fisher's exact test), consistent with Hypothesis 2.

Figure 2: Choices made in Treatments 3 and 4

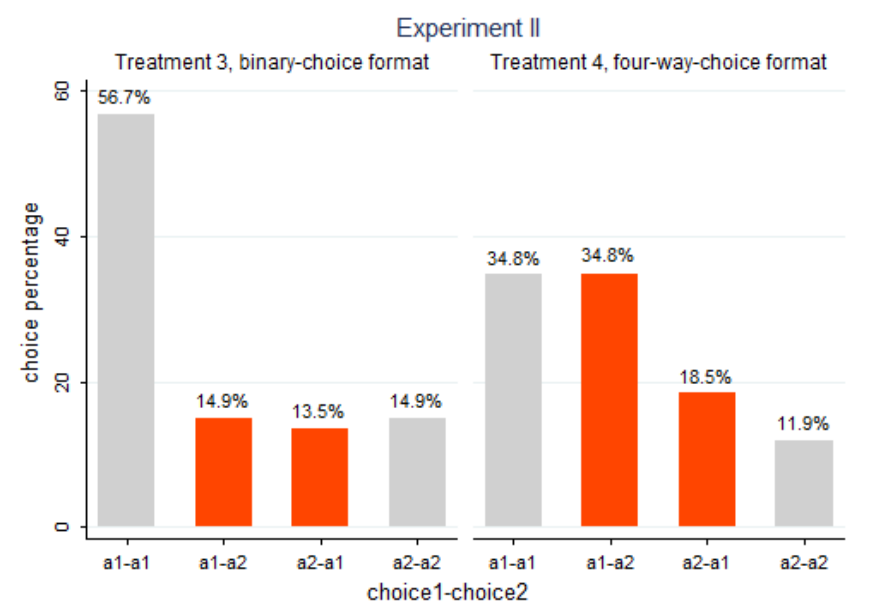

RESULT 2: When given the explicit opportunity to choose a lottery between two alternatives, more than half of participants choose it. Lotteries are chosen significantly more often than in an economically equivalent repeated-choice format where only indirect randomization is possible.

Once again, random utility perturbations cannot explain the data of Experiment II as we 
observe a pure framing effect.

\subsection{Experiment III: Reversals between longer preference lists}

In Experiments I and II, there are only two alternatives among which participants can choose. This differs from many real-life settings such as university choice where decision-makers rank more than two alternatives. Experiment III examines whether reversals in the rank order can also be found in choices between longer lists. To control for beliefs about the uncertain environment, we again employ a simple experiment with vouchers (or cash) as prizes. In addition, Experiment III involves an unannounced second part to test the implications of Proposition 2 for the present setting.

\subsubsection{Design}

In each of two experiments, a classroom experiment and a newspaper experiment, there are four available prizes, $a_{1}$ to $a_{4}$, consisting of one or two gift vouchers and/or cash. Table 1 shows the prizes for both experiments.

Table 1: Prizes to be ranked in the experiments

\begin{tabular}{lll}
\hline \hline & Classroom & Newspaper \\
\hline$a_{1}$ & 19 Euro Starbucks voucher & 54 Euro Amazon voucher \\
$a_{2}$ & 19 Euro Amazon voucher & 42 Euro in cash \\
$a_{3}$ & 14 Euro Starbucks \& 10 Euro Amazon vouchers & 34 Euro Amazon voucher \& 12 Euro in cash \\
$a_{4}$ & 8 Euro Starbucks \& 16 Euro Amazon vouchers & 14 Euro Amazon voucher \& 30 Euro in cash \\
\hline \hline
\end{tabular}

Monetary prize values in the newspaper experiment are higher than in the classroom experiment in order to motivate the newspaper readers to participate. To participate, they $\log$ on to a webpage and submit their preferences. Since readers of the WZB-Mitteilungen may be less likely to go to Starbucks than students, we replace the Starbucks vouchers with cash payments.

Participants are asked to rank the prizes on two lists, list (i) and list (ii), where each 
list is chosen to be payoff-relevant with a probability of 0.5 . Each list, if chosen to be payoff-relevant, delivers the differently ranked prizes with given probabilities. This yields an incentive-compatible mechanism to report the full preference ranking: on both lists, the prize ranked first is received with the highest probability, the prize ranked second with the second highest probability, etc. As shown in Table 2 , the distribution of probabilities among the different ranks slightly differs across the two lists.

Table 2: Probabilistic consequences of the two preference lists

\begin{tabular}{lll}
\hline \hline Probability of getting prize ranked & List (i) & List (ii) \\
\hline 1st & .5 & .4 \\
2nd & .3 & .3 \\
3rd & .2 & .2 \\
4th & 0 & .1 \\
\hline \hline
\end{tabular}

An expected-utility maximizer with strict preferences over the available prizes $a_{1}$ to $a_{4}$ would list her most preferred prize first on both lists, the second preferred prize second etc. Any other combination of lists puts avoidable probability mass on receiving a less desirable outcome. Following Proposition 1, a responsibility averter may decide to submit different lists 21

To test for a specific implication of responsibility aversion, we add to the experiment a (surprise) second part that appears immediately after the participants submit their preferred lists. In the second part, participants simply pick their most preferred prize $\left(a_{1}, a_{2}, a_{3}\right.$ or $\left.a_{4}\right)$. (Only one of the two parts was payoff-relevant, as detailed below.) The choice in the second part does not allow for randomization and we thus interpret it as the most preferred option according to the reference preference $\succsim$. Following Proposition 2, we predict a systematic pattern: a responsibility averter who top-ranks two different prizes on list (i) and list (ii) in the first part of the experiment will list the $\succsim$-optimal prize on list (i), not list (ii), because the probability of receiving the top-ranked prize is higher on list (i) than on list (ii). Therefore,

\footnotetext{
${ }^{21}$ This requires, however, a particular understanding of responsibility aversion: the fact that the implemented rank is chosen randomly does not (fully) relieve the decision-maker from her desire to avoid responsibility. To the extent that the random choice of ranks reduces the responsibility attitudes, the rank reversals may be reduced.
} 
we hypothesize that a majority of participants in the subset of participants who top-ranked two different prizes on list (i) and list (ii) reports a choice in the second part that confirms the top rank of list (i), not list (ii).

HYPOTHESIS 3: Responsibility averters might submit different choice lists (i) and (ii) in the first part of the experiment. Among participants who list different prizes on rank 1 of lists (i) and (ii), a majority will confirm list (i) in the second part of the experiment.

In the classroom experiment, 10 percent of participants receive a prize in the first part of the experiment and a different set of 10 percent of participants receive a prize in the second part. In the newspaper experiment, there was an ex-ante fixed number of 10 winners for the first part and 10 different winners for part II, to be drawn randomly.

\subsubsection{Results}

In the classroom experiment, a total of 314 students participated. One hundred and twentysix of them (40\%) stated different preferences on list (i) and list (ii). Almost all reversals $(92 \%)$ involve adjacent ranks, indicating a systematic nature of the reversals: in most cases of reversals, a participant's list (ii) choice simply switches the ranking of two prizes that are next to each other on the same participant's list (i). Furthermore, 99 out of the 126 reversals $(79 \%)$ occurred at the first rank of the lists. Confirming Hypothesis 3, we observe that of these 99 occurrences, 56 participants confirm list (i) in the experiment's second part, 30 confirmed list (ii), and 13 participants confirmed neither list (i) nor list (ii). The difference in confirmations of list (i) and list (ii) is significant $(p<0.01$, one-sided Binomial test with $N=86) .{ }^{22}$

In the newspaper experiment, 194 participants submitted a complete set of online re-

\footnotetext{
${ }^{22}$ Note that alternatives here are trichotomic: confirm list (i), confirm list (ii), confirm none of the two lists. However, a Binomial test (on confirming list (i) versus confirming list (ii)) can still be used if the third group (that does not confirm any list) is made up 50-50 by the two former groups in expectation. In that case, the number of observations relevant for the test equals the total number of reversals less the number of participants not confirming any list. Since there is no reason to assume that the third group is selective, this is how we proceed. We therefore have $N=99-13=86$.
} 
sponses ${ }^{23}$ Of these, 29 participants $(15 \%)$ do not submit identical lists. This percentage is lower than in the classroom experiments, but the nature of reversals is similar. We observe that in 28 out of 29 reversals $(97 \%)$ only adjacent ranks were involved. Of the 29 reversals, 15 occurred at the first rank (52\%). We also find supportive evidence regarding Hypothesis 3's prediction that choices in the experiment's second part tend to confirm the first choice on list (i). In the second part, 10 participants confirmed list (i) and only 3 confirmed list (ii). This difference is statistically significant ( $p<0.05$, one-sided Binomial test with $N=13$ ).

RESULT 3: 40\% of participants in the classroom experiment and 15\% of participants in the newspaper experiment submit different rank-order lists. Participants who show reversals at rank 1 between their two lists show a systematic pattern in that they tend to confirm their first choice of list (i) in the second part of the experiment.

\section{Empirical evidence: Reversals in university applications}

In this section we report real life evidence on an important decision in people's lives: university applications. We show that the results of the stylized experiments carry over to this context, in the sense that a non-negligible amount of induced randomness occurs. We also discuss allocative consequences of these decisions.

Admissions to German undergraduate university programs in the medical subjects are centrally administered by a clearinghouse. The clearinghouse assigns applicants according to the following three procedures that are implemented in a sequential order:

(1) Procedure A admits students who are top of the class to up to $20 \%$ of seats.

(2) Procedure $\mathrm{W}$ admits students with long waiting times to up to $20 \%$ of seats.

(3) Procedure U represents admission by universities according to their own criteria to the

\footnotetext{
${ }^{23}$ About half of the participants were directly affiliated with WZB and thus either social scientists or familiar with research in social sciences.
} 
remaining (at least $60 \%$ of) seats.

For each of the three procedures, applicants are asked to submit a preference ranking of universities, which may either be identical or different across procedures. All rank-order lists are submitted at the same moment in time. The central clearinghouse employs the three procedures in a strictly sequential order: all applicants who are matched in procedure A are firmly assigned a seat at their matched university and do not take part in subsequent procedures. All remaining applicants enter procedure W. Likewise, after procedure W, all applicants who are still unmatched enter procedure U. The fact that applicants can submit three potentially different rank-order lists of universities, each of which may be relevant, is a unique property of the German mechanism and makes it suitable for our analysis.

Because applicants who are successful in procedure $\mathrm{W}$ are a disjoint group with relatively poor grades and low chances of being admitted through the other two procedures, we restrict our attention to procedures $\mathrm{A}$ and $\mathrm{U}$, where admission largely follows the grade point average (GPA) in final secondary school examinations. For both procedures A and U, applicants are allowed to rank no more than six universities 24 In the following subsection, we briefly introduce the two procedures and the relevant matching literature.

Subsection 4.3 assesses the frequency of preference reversals, i.e., the submission of different preference lists by the same applicants. As we will explain in detail, not all instances of self-contradicting applications violate standard theory, due to strategic reasons. We therefore need to identify a subset of cases where these strategic reasons do not apply. Using the assumptions on responsibility attitudes in Section 2, our empirical strategy is as follows. We first establish that in the absence of responsibility aversion, applicants have no incentive to distort their rank-order list for procedure $\mathrm{U}$. That is, the ranking in procedure $\mathrm{U}$ contains an applicant's preference $\succsim$ under the hypothesis that responsibility aversion is absent. (In

\footnotetext{
${ }^{24}$ For a more detailed description of the admission process see Braun et al. (2010), Braun and Dwenger (2009) and Westkamp (2013).
} 
fact, the information brochure of the clearing house indicates to applicants that it is in their interest to reveal their preferences truthfully under procedure U. We confirm this below by providing theoretical arguments.) We then establish that we can identify a subset of applicants who have strict incentives to also report the same rank-order list under procedure A: these are applicants for whom a preference reversal may not only have a material consequence but who also have no strategic incentives to game the application system (see Subsections 4.3.2 and 4.3.3. Within this subgroup of applicants, we can thus identify responsibility averse decision-makers as those who reverse their two rank-order lists between procedures $\mathrm{A}$ and $\mathrm{U}$. A reversal by these individuals increases their probability of ending up at a suboptimal university. Notice that by construction of our argument, this is a violation of the assumption of responsibility neutrality/loving, which is needed to establish the preference list submitted for procedure $\mathrm{U}$ as the true preference ordering. We therefore cannot assume that the rankorder lists of procedure U reflect the true preferences of these applicants. But calculations in Subsection 4.3.4 show that no matter what is the true preference order, the consequences of the preference reversals are economically significant.

\subsection{Matching procedures}

\subsubsection{Procedure U}

For expositional reasons, we start by introducing procedure $\mathrm{U}$ even though it is administered at the end of the admission process. Procedure U corresponds to a two-sided market where not only applicants but also universities express their preferences over their possible matches. Each university ranks the applicants who list it on their preference list, using the final grade from school as the predominant but not necessarily the only criterion to discriminate between applicants. Given the preference lists of universities and applicants, the central clearinghouse applies the college-proposing Gale-Shapley algorithm. The algorithm was first described by 
Gale and Shapley (1962) although similar ideas had been in use since the 1950s in the U.S. clearinghouse for the first jobs of doctors (see Roth (2008)). A description of the collegeproposing Gale-Shapley mechanism is in the appendix.

As indicated above, the information brochure of the clearinghouse explicitly advises applicants to reveal their true preferences in procedure $\mathrm{U}$. This advice is justifiable by two theoretical arguments. First, in the college-proposing Gale-Shapley mechanism all successful manipulations can also be accomplished by truncations, see Roth and Peranson (1999), p.762, referring to results by Roth and Vande Vate (1991). Thus, even if applicants strategically truncate their lists submitted in procedure $\mathrm{U}^{25}$ the correct rank order of the remaining choices is preserved. It is also noteworthy that truncations require the least information about others' preferences among all possible manipulations that are potentially beneficial to the decision-maker (see Roth and Rothblum 1999).

Second, if preferences of universities are perfectly correlated (which they are if universities rank applicants only by their final grades from school), then there is only one stable matching ${ }^{26}$ In this case, the stable matching is achieved by both the college- and the studentproposing Gale-Shapley mechanism. Since the latter is strategy-proof (see Roth 1982), it then follows that truth-telling is also a dominant strategy in the college-proposing Gale-Shapley mechanism 27

Thus, we can conclude that the incentives to misrepresent one's preferences in procedure U are null for perfectly correlated preferences of universities and they are small if the preferences

\footnotetext{
${ }^{25}$ In the information brochure, the central clearinghouse does not mention the possibility of such truncations or other forms of strategically misrepresenting one's true preferences in procedure U (except for a "pre-selection" stage which does not affect our analysis).

${ }^{26}$ In a stable matching everybody prefers their match over no match at all and there is no applicant and university who are not matched but who would both prefer to be. To see why there is only one stable matching if university preferences are perfectly correlated, note that in any stable matching the applicant ranked highest by the universities gets his preferred matching. Thus, there is only one stable matching for the applicant with the best final grade. This argument can be repeated for all other applicants.

${ }^{27}$ Another potential reason for misrepresenting one's preferences is the fact that only up to six universities can be ranked. This can induce applicants to include safe options (Haeringer and Klijn 2009). However, we will restrict attention to top students (restriction II) who do not face the risk of being unassigned to a seat at one of their six choices.
} 
of universities are strongly correlated. This is the case in the German university admission system where all universities have to use the GPA as the main criterion due to legal constraints and where some universities even base their ranking of applicants solely on the final grade. Even if small incentives to misrepresent preferences exist, then truncations of the true preference list are optimal and far more plausible than other manipulations (especially given the clearinghouse's advice). We will therefore consider the non-empty parts of the rank-order lists submitted under procedure $\mathrm{U}$ as reflecting the true preferences of applicants, under the null hypothesis of responsibility neutral or responsibility loving decision-makers.

\subsubsection{Procedure A}

Procedure A is employed to reward excellent GPAs in secondary schools. Applicants with the best average grades are selected and then assigned according to the preference list that they submit for procedure A. To this end, the central clearinghouse applies the so-called Boston mechanism, which assigns as many applicants as possible to their first choice ( $k^{\text {th }}$ choice) and considers second choices $\left((k+1)^{t h}\right.$ choices $)$ only if there are still seats left at the end of the first $\left(k^{t h}\right)$ round (cf. the appendix for details on the Boston mechanism) ${ }^{28}$

The Boston mechanism implies that an applicant ranking a university in $k^{\text {th }}$ position is admitted before applicants ranking a university in $(k+1)^{t h}$ position are considered - independently of her high-school GPA. Hence, it may be advantageous for some applicants to manipulate their true preference ordering by skipping a university if they do not have any chance of getting a seat. We need to take this incentive into account when looking for deliberate reversals in the preference lists.

In addition to the strategic incentives due to the Boston mechanism itself, the sequential nature of the admission process creates incentives to misrepresent one's preferences in proce-

\footnotetext{
${ }^{28}$ For a systematic description of the mechanism see Abdulkadiroglu and Sönmez (2003).
} 
dure A. Procedure A is the first to be administered. As assignments in each of the procedures are fixed, only applicants who have not been admitted through procedure A (or through procedure $\mathrm{W}$ ) participate in procedure $\mathrm{U}$. Both procedures A and $\mathrm{U}$ largely follow average grades, that is, applicants with a very good GPA have a chance of being admitted in both procedures. These individuals should avoid being matched to a less preferred university in procedure A and wait for procedure $\mathrm{U}$ where they have a very good chance of being admitted to one of their top choices. Applicants might therefore submit rank-order lists of universities in procedure A that are shorter than their true preference order 29

In our analysis below, we eliminate all cases where preference reversals can be driven by the above-described strategic motives. The hypothesized motive of responsibility aversion would still apply to the data context, as the applicants can seek randomness by submitting different lists.

HYPOTHESIS 4: Responsibility averters may use the two preferences lists for procedure $A$ and procedure $U$ to invoke a random determination of the outcome. After elimination of applicants with strategic reasons to submit different lists, significant proportions of applicants may still submit different rank-order lists for the two procedures.

\subsection{Description of the data}

We use the (anonymized) information collected by the central clearinghouse covering seven waves of applications between winter term 2005/06 and winter term 2008/09. During our observation period the following six subjects were centrally administered and are part of our data set: biology, medicine, pharmacy, psychology, animal health, and dentistry. The data set contains all applications for these subjects and records all information provided by

\footnotetext{
${ }^{29}$ Braun et al. (2010) demonstrate that many applicants react insufficiently to this incentive and submit too long lists for procedure A. The objective of procedure A-to give an additional advantage to the applicants with highest GPA-therefore fails to be met.
} 
the applicants including data on individual characteristics such as final GPA, age, sex, and location. Most important for our purpose, the database provides information on the admission procedures that a prospective student has participated in as well as his or her rank-order lists on universities for the different procedures.

To avoid multiple counting, we only consider each individual's first application in the data set. After excluding individuals not applying for procedure $U$ and discarding individuals whose preference list for procedure $\mathrm{U}$ might be incomplete due to pre-selection, we are left with a total number of 224,016 first-time applications ${ }^{30}$

\subsection{Results: Preference reversals in university applications}

\subsubsection{Full data set}

We first examine the full set of 224,016 first-time applicants to study whether preference lists coincide in procedures A and U. Table 3 shows where the entries on the lists submitted for procedure $\mathrm{U}$ appear as entries for procedure A. Row $i, i=1, \ldots, 6$, of the table contains, for the universities on rank $i$ for procedure $\mathrm{U}$, the percentage of cases in which the same applicant listed the university on rank $j$ of the list for procedure A.

The diagonal elements in the table indicate unchanged preferences. For example, in $75 \%$ of applications the top-ranked university from procedure $\mathrm{U}$ is also ranked first in procedure $\mathrm{A}$. Similarly, $63 \%$ of universities ranked second in procedure $\mathrm{U}$ are ranked second in procedure A, etc. The off-diagonal elements report the discrepancies between the lists that applicants

\footnotetext{
${ }^{30}$ All procedures are two-stage procedures. At the first stage applicants are "pre-selected" (in the language used by the clearing house) and at the second stage the pre-selected applicants compete for admission to one of their preferred universities. Universities can delegate pre-selection to the clearinghouse, which in this case shortlists applicants according to the preference rank the applicant has given to the university and according to GPA. The pre-selection criteria applied by the universities differ, requiring for example that the university be listed as a first or first to third preference. Some universities use a combination of average final grade and preference rank. Importantly, after pre-selection the applicants are allowed to reshuffle the ordering of their rank-list. We only consider the final rank-order list for procedure $U$ as this list should be free from strategic manipulations.
} 
Table 3: Conditional proportions, showing where universities ranked in procedure U appear in rank-order lists for procedure A (all entries in \%)

\begin{tabular}{c|rrrrrrr|r}
\hline \hline $\mathrm{U} / \mathrm{A}$ & 1 & 2 & 3 & 4 & 5 & 6 & not ranked & total \\
\hline 1 & 75.43 & 4.05 & 1.91 & 1.14 & 0.78 & 0.54 & 16.15 & 24.57 \\
2 & 5.34 & 63.40 & 4.83 & 2.37 & 1.64 & 1.10 & 21.33 & 37.48 \\
3 & 2.71 & 5.23 & 58.80 & 4.76 & 2.84 & 1.78 & 23.89 & 41.20 \\
4 & 2.35 & 3.45 & 5.77 & 55.88 & 4.94 & 2.64 & 24.96 & 44.12 \\
5 & 1.74 & 2.36 & 3.42 & 5.88 & 55.23 & 4.59 & 26.80 & 44.77 \\
6 & 1.66 & 1.89 & 2.36 & 3.52 & 5.45 & 54.92 & 30.20 & 45.08 \\
\hline \hline
\end{tabular}

Notes: Full data set as described in Section 3.2 ( $N=224,016$ observations).

Source: Own calculations based on ZVS data on applicants, waves 2005/06 to 2008/09.

submit in the two procedures A and U. Differences at a certain preference rank can result either from listing different universities or from not listing any university on a given rank of one list but listing a university on the same rank of the other list. The results in Table 3 show that a considerable number of subjects submit different lists. Every fourth applicant has different entries on the top rank of her two lists. The entries directly above or below to the diagonal indicate the following pattern: many preference reversals are formed by moving a university to an adjacent preference rank.

Notice that skipping the top-ranked university in procedure A moves up all universities named on rank 2 and below. In Table 3 skipping the top-ranked university is therefore recorded in multiple preference reversals that appear further down the list. To avoid such double counting, Table 4 repeats the counting exercise of Table 3 but only includes the first preference reversal within an application. Entries in rows 2...6 of Table 4 (marked with an asterisk) reflect additional cases of preference reversals, relative to previous ranks. For example, not ranking the top U-ranked university in procedure A only appears in the Table's first row as "not ranked" while the effects of this reversal further down the list are not counted. Table 4 therefore counts each application with a preference reversal exactly once. It shows that preference reversals are widespread: a total of $64 \%$ of all applicants reverse at least one preference rank between procedures $\mathrm{U}$ and $\mathrm{A}$ or do not list a university in procedure $\mathrm{A}$ that is listed in procedure U. Disregarding reversals from not ranking universities in procedure A 
but listing them in procedure $\mathrm{U}(36 \%)$ shows that approximately $29 \%$ of applicants change the ordering of their preference lists.

Notes: See Table 3 .

\begin{tabular}{c|rrrrrrr}
\multicolumn{7}{c}{ Table 4: Preference reversals (all entries in \%) } \\
\hline \hline U / A & 2 & 3 & 4 & 5 & 6 & not ranked & total \\
\hline 1 & 4.05 & 1.91 & 1.14 & 0.78 & 0.54 & 16.15 & 24.57 \\
$2^{*}$ & - & 4.58 & 1.92 & 1.16 & 0.78 & 7.67 & 16.11 \\
$3^{*}$ & - & - & 3.55 & 1.58 & 0.86 & 3.89 & 9.88 \\
$4^{*}$ & - & - & - & 2.87 & 1.12 & 3.02 & 7.01 \\
$5^{*}$ & - & - & - & - & 1.80 & 2.21 & 4.01 \\
$6^{*}$ & - & - & - & - & - & 2.86 & 2.86 \\
total & 4.05 & 6.49 & 6.61 & 6.39 & 5.09 & 35.81 & 64.44 \\
\hline \hline
\end{tabular}

RESULT 4: Overall, 64\% of university applicants submit two different rank-order lists in procedures $A$ and $U$.

\subsubsection{Restriction I: Ruling out strategic manipulations}

As noted in Section 4.1.2, applicants may have incentives to skip a university in the list for procedure A if they expect not to have a chance of being admitted at that university. They may also truncate their rank-order list for procedure A in order to avoid being matched to a lower preference in procedure A and instead wait for procedure U. In order to identify these incentives, applicants need to anticipate their chances of success for procedure A. The clearinghouse publishes detailed information on the application characteristics of admitted candidates for every university-field combination in each year. It also advises applicants to use the historical admissions statistics when devising their list for procedure A, and it points out the potential advantage of manipulating the list. Under the auxiliary assumption that admission chances are stable from year to year (which is close to correct), the applicants have the information necessary for strategic manipulations and they will plausibly use it. To exclude all observations where strategic considerations of the applicants can be at work, summarized as "restriction I", we first select all applicants who, on the considered rank, would have been admitted in procedure $\mathrm{A}$ to the university listed in procedure $\mathrm{U}$, in the preceding 
year. Second, we restrict attention to ranks where a university is listed in both procedures to exclude strategic truncations. This restricts the analysis to 91,485 first-time applications.

Table 5: Conditional proportions after restriction I, ruling out strategic incentives (all entries in \%)

\begin{tabular}{c|rrrrrrr|r}
\hline \hline $\mathrm{U} / \mathrm{A}$ & 1 & 2 & 3 & 4 & 5 & 6 & not ranked & total \\
\hline 1 & 87.09 & 4.99 & 2.43 & 1.44 & 1.00 & 0.70 & 2.36 & 12.91 \\
2 & 6.51 & 71.39 & 6.46 & 3.19 & 2.36 & 1.59 & 8.50 & 28.61 \\
3 & 2.96 & 6.03 & 66.01 & 6.20 & 4.03 & 2.49 & 12.29 & 33.99 \\
4 & 3.10 & 3.92 & 6.73 & 62.10 & 6.47 & 3.67 & 14.01 & 37.90 \\
5 & 1.95 & 2.64 & 3.89 & 6.51 & 63.01 & 5.99 & 16.02 & 36.99 \\
6 & 1.72 & 2.19 & 2.95 & 4.39 & 6.24 & 61.88 & 20.65 & 38.12 \\
\hline \hline
\end{tabular}

Notes: We only consider applications where both preference rankings contain a nonempty cell in the considered rank, and where the applicant's entry in procedure U would have been successful in the previous year, conditional on previous ranks being unsuccessful. $N=91,485$ observations.

Source: Own calculations based on ZVS, data on applicants, waves 2005/06 to 2008/09.

In Table 5, the fraction of applications stating a university on the same rank in procedure A as in procedure $\mathrm{U}$ increases: $87 \%$ compared to $75 \%$ (Table 3) of applicants top-rank the same university in procedures $\mathrm{U}$ and $\mathrm{A}$. In particular, the proportion of universities that are top-ranked in procedure $\mathrm{U}$ but not ranked in procedure A drops significantly from $16 \%$ (Table 3) to $2 \%$. This is exactly what we would expect if applicants strategically skip their first preference in procedure A because they do not expect to have a chance of getting a seat. On lower ranks, the difference in the proportions of applicants not ranking a university in procedure A named in procedure $\mathrm{U}$ is still significant but smaller than in the top ranks. This, too, is to be expected from rational applications, as the Boston Mechanism's strategic incentives to skip a university are greater for top ranks. The evidence is generally consistent with the hypothesis that part of the preference reversals found in Tables 3 and 4 are due to strategic considerations of the applicants. Yet, after applying restriction I, we still see a large proportion of individuals reversing their rank-order lists. Table 6 summarizes the results of Table 5 without double counting further down the list (analogous to the transition from Table 3 to Table 4). It shows that almost $28 \%$ of applicants in procedure A either do not rank a university listed in procedure $\mathrm{U}(7 \%)$ or swap preferences $(21 \%)$. 
Table 6: Preference reversals after restriction I, ruling out strategic incentives (all entries in $\%)$

\begin{tabular}{c|rrrrrrr}
\hline \hline $\mathrm{U} / \mathrm{A}$ & 2 & 3 & 4 & 5 & 6 & not ranked & total \\
\hline 1 & 4.99 & 2.43 & 1.44 & 1.00 & 0.70 & 2.36 & 12.91 \\
$2^{*}$ & - & 3.22 & 1.38 & 0.85 & 0.55 & 1.72 & 7.72 \\
$3^{*}$ & - & - & 1.64 & 0.73 & 0.41 & 1.02 & 3.80 \\
$4^{*}$ & - & - & - & 0.96 & 0.40 & 0.60 & 1.96 \\
$5^{*}$ & - & - & - & - & 0.45 & 0.39 & 0.84 \\
$6^{*}$ & - & - & - & - & - & 0.47 & 0.47 \\
total & 4.99 & 5.65 & 4.45 & 3.53 & 2.51 & 6.57 & 27.70 \\
\hline \hline
\end{tabular}

Notes: See Table 5

RESULT 5: After removing applicants who have an incentive to submit different rank order lists, 28\% of applicants display preference reversals between lists. $13 \%$ of applicants submit pairs of preference lists where a different university appears on rank 1.

\subsubsection{Restriction II: Ruling out irrelevance}

When submitting their preference lists, applicants do not know for certain whether they will be selected for any of the procedures. But by using the historical admissions data, they can assess the probability with which each of the procedures applies to them. A natural possibility is that applicants sometimes do not follow their true preferences when filling out lists in procedures that are most likely not relevant for them. This may result in preference reversals that are solely due to the fact that one list is likely to be irrelevant: e.g., an applicant who can be sure of being matched in procedure A (because her GPA is clearly good enough for her top choice on list A) might as well report a reversed list for procedure U. To rule out irrelevance of procedure U ("restriction II"), we drop individuals from the data set obtained after applying restriction I whose GPA is at least 0.4 grade points better than the threshold needed for admission on the considered rank of list A. Further, we focus on applicants who belong to the (top 20\%) group that is selected for procedure A to make sure that the preference list for $\mathrm{A}$ is relevant, too.

Only considering the 8,508 applicants who have good chances of being admitted in both 
Table 7: Conditional proportions after restriction I and II, ruling out strategic incentives and irrelevance (all entries in \%)

\begin{tabular}{c|rrrrrrr|r}
\hline \hline $\mathrm{U} / \mathrm{A}$ & 1 & 2 & 3 & 4 & 5 & 6 & not ranked & total \\
\hline 1 & 93.67 & 3.39 & 0.89 & 0.38 & 0.27 & 0.15 & 1.26 & 6.34 \\
2 & 1.90 & 74.76 & 3.83 & 1.29 & 0.65 & 0.33 & 17.23 & 25.24 \\
3 & 0.82 & 3.35 & 66.14 & 3.42 & 2.05 & 0.90 & 23.27 & 33.86 \\
4 & 0.64 & 1.93 & 3.80 & 61.52 & 3.62 & 1.50 & 26.99 & 38.48 \\
5 & 0.23 & 0.46 & 1.67 & 3.96 & 61.13 & 3.08 & 29.48 & 38.87 \\
6 & 0.30 & 0.30 & 0.80 & 1.89 & 2.98 & 59.32 & 34.41 & 40.68 \\
\hline \hline
\end{tabular}

Notes: In addition to restriction I, we discard applicants who do not belong to the top $20 \%$ group selected for procedure A or individuals whose GPA is at least 0.4 grade points better than the threshold needed for admission on the considered rank of list $\mathrm{A}$, or for any university listed on a previous rank (restriction II). $N=8,508$ observations.

Source: Own calculations based on ZVS data on applicants, waves 2005/06 to 2008/09.

procedures $\mathrm{A}$ and $\mathrm{U}$ and who are not prone to strategic behavior, we find that the percentage of individuals stating congruent preferences in both procedures increases. $94 \%$ of applicants top-rank the same university in procedures $\mathrm{U}$ and $\mathrm{A}$ (Table 7); the remaining $6 \%$ either do not rank their top university from procedure $\mathrm{U}$ at all or state it on a lower rank in procedure A. In order to determine the number of individuals reversing at least one preference, we again exclude double-counting and focus on the first preference reversal within each application. The resulting Table 8 shows that, after restricting our data set to rule out strategic behavior and carelessness of the applicants, $14 \%$ of all individuals in our data set submit preference lists for procedures $\mathrm{A}$ and $\mathrm{U}$ that differ in at least one rank.

Table 8: Preference reversals after restrictions I and II, ruling out strategic incentives and irrelevance (all entries in \%)

\begin{tabular}{c|rrrrrrr}
\hline \hline $\mathrm{U} / \mathrm{A}$ & 2 & 3 & 4 & 5 & 6 & not ranked & total \\
\hline 1 & 3.39 & 0.89 & 0.38 & 0.27 & 0.15 & 1.26 & 6.34 \\
$2^{*}$ & - & 2.09 & 0.65 & 0.32 & 0.18 & 0.87 & 4.10 \\
$3^{*}$ & - & - & 0.84 & 0.46 & 0.12 & 0.48 & 1.89 \\
$4^{*}$ & - & - & - & 0.49 & 0.15 & 0.33 & 0.98 \\
$5^{*}$ & - & - & - & - & 0.28 & 0.31 & 0.59 \\
$6^{*}$ & - & - & - & - & - & 0.24 & 0.24 \\
total & 3.39 & 2.99 & 1.86 & 1.54 & 0.88 & 3.48 & 14.13 \\
\hline \hline
\end{tabular}

Notes: See Table 7

RESULT 6: In a conservative estimate, we find that 14\% of applicants submit preference lists that differ in at least one rank across procedures. These choices are consistent with responsibility aversion and can neither by explained by the characteristics of the application 
procedure nor by irrelevance of one of the lists for the applicants.

\subsubsection{Consequences arising from preference reversals}

As our final set of results, we report the frequencies with which applicants who reverse their preferences between lists are matched unnecessarily to suboptimal universities. For this exercise, we restrict attention to applicants who are still in the data set after both restriction I and restriction II and ask: would the applicants who show preference reversals be matched to a better university if they were to report the same rank-order list for both procedures?

Since these applicants reverse their preference orders between the two procedures, it is not obvious by which preference order one should evaluate their outcome. We thus consider both reported preference lists as candidates for the "true" preference order. First, we consider the case that applicants report their true preferences under procedure U. For the first three of the seven waves of applications, we have the necessary data available to answer the question 31 Of the $N=5,170$ applications that survive restriction I and restriction II and that appear in these three waves, $757(15 \%)$ show preference reversals between the two lists, which is comparable to our previous results that use all data. Column (1) of Table 9 shows the preference rank on the U-list that was realized for these applicants, and column (2) displays which rank they could have obtained if they had reported their U-ranking also under procedure A.

The comparison of columns (1) and (2) shows that preference reversals substantially harm the individuals who commit them. While only $66.1 \%$ of applicants in this group actually obtain their most preferred university seat, $86.9 \%$ could have obtained their first choice under the counterfactual that they simply report their U-list also in A. The difference is 20.8 percentage points or a total of 158 applicants in this restricted sample 32 Column (3) shows that

\footnotetext{
${ }^{31}$ For the remaining waves, the matching outcome is not available in our data set.

${ }^{32}$ The inefficiencies are even stronger if we consider an alternative counterfactual strategy where the applicants use their preference list for procedure U but truncate it optimally. Instead of $86.9 \%$ as reported in column (2) of Table 9 a total of $93.1 \%$ of the same 757 applicants could have obtained their top choice.
} 
Table 9: Matching outcomes (all entries in \%)

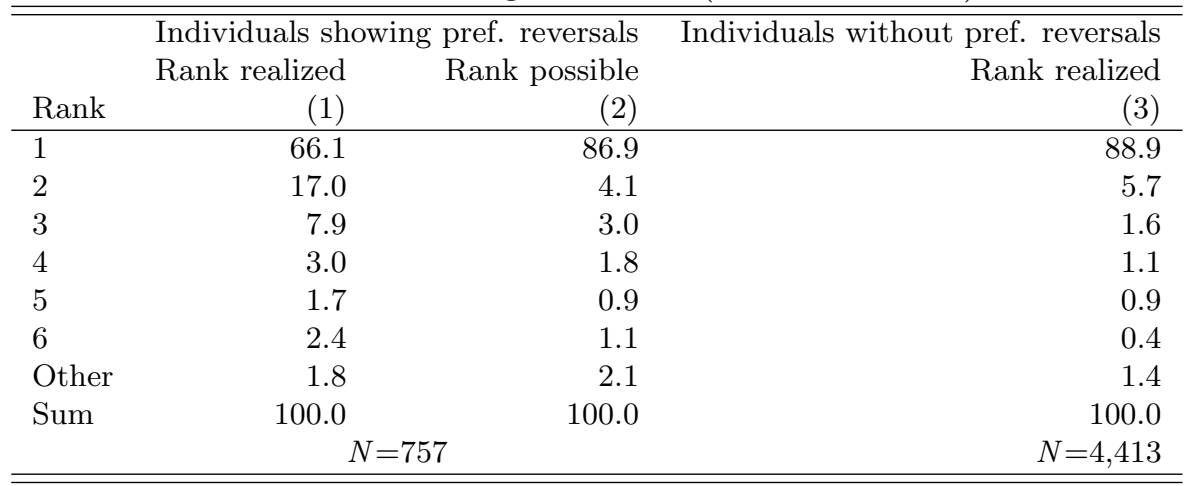

Notes: Matching outcomes of $N=5,170$ applicants in three waves. All entries in percent. (1) contains actual matches of applicants who submitted lists containing preference reversals between A and U. (2) contains hypothetical matches under the counterfactual assumption that the same set of applicants submit the list for $\mathrm{U}$ under both procedures. (3) contains the actual matches of all applicants who submitted consistent lists.

Source: Own calculations based on ZVS data on applicants, waves 2005/06 to 2006/07.

the remaining set of 4,413 applicants who submit consistent lists in A and U have success rates that are very close to the counterfactual results of column (2). This indicates that the two sets of applicants on average face decision problems of comparable importance.

Separate calculations show that the inefficiency becomes even stronger if we further restrict the sample to the 190 applicants (again applying restrictions I and II in the three relevant waves) who simply flip their top two choices between the lists A and U. Of them, only $53.7 \%$ actually obtained their top U-ranked choice, whereas $82.1 \%$ could have obtained it under the counterfactual strategy of submitting the U-list to both procedures.

We now consider the case that the rank-order list submitted under procedure A reflects the "true" preference order. Under this assumption, distorting one's preference list under procedure $\mathrm{U}$ is even more harmful in our sample: all applicants who are eligible for procedure $\mathrm{A}$ have a very high chance to be matched to their top A-ranked university under procedure U. A further data restriction is, however, that the central clearinghouse itself implements procedure $\mathrm{U}$ only for a subset of universities and we thus only have outcome data for procedure $\mathrm{U}$ on this subset of universities. We can therefore construct the required counterfactual outcomes 
for only 3,162 individuals who satisfy restrictions I and II, whereof 414 applicants (13\%) display preference reversals. For these individuals, the possible rate of being matched to their top A-ranked university under procedure $\mathrm{U}$ is $95.7 \%$. The applicants' actual success rate of being matched to their top A-ranked university is only $65.9 \%$. The difference in actual versus possible outcome is therefore even larger than in Table 9. We note that this subsample of universities may create a selection problem for this calculation. We also note that, importantly, it is more plausible that applicants report their true preference under procedure $\mathrm{U}$ than under procedure A because the clearinghouse advises them to do so.

RESULT 7: Preference reversals have allocative consequences. Considering only applicants for whom both lists are relevant but who have no strategic incentives to submit lists with preference reversals, more than $20 \%$ could have been allocated to their top-ranked university seat but received a different seat as a consequence of their reversal.

\section{Conclusion}

The paper formulates a theory of responsibility attitudes, following the psychological literature on regret aversion and indecision, and documents a revealed preference for randomization in a number of different contexts. First, across several experiments we observe that a significant fraction of players choose to randomize between options. Many of the observed choices to randomize appear to be deliberate, in the sense that they follow a systematic pattern that our theory of responsibility attitudes predicts. The observed desire for randomization is robust to variations of the framing: it appears not only when the experimental participants have to make several choices and only one of them becomes relevant with a certain probability, but also when there is the possibility to choose a lottery directly. These direct or indirect randomization choices violate most standard models of choice under uncertainty. Second, in a large data set on university applications in Germany, a pervasive pattern of reversals 
of preferences between rank-order lists appears and induces avoidable randomness. In this context, significant economic consequences arise from the preference reversals.

We have identified a procedural preference that to our knowledge has not yet been studied empirically in economics, apart from studies on procedural fairness. Our proposed explanation for the choice of randomization is fear of regret from taking the wrong decision. The decisionmaker feels less regret if an ex post bad choice is due to a random outcome. The motives to avoid a decision may well be different between the different data contexts, as it seems natural that different kinds of potential regrets play a role when deciding between universities versus when deciding between vouchers. The experiments clearly cannot tell the story of why university applicants leave an important decision to chance. But they can add to the picture by providing evidence that preference reversals and their implied violations of standard choice theory are sometimes chosen deliberately and not by accident. 


\section{References}

Abdulkdadiroglu, Atila and Tayfun Sönmez. 2003. School Choice: A Mechanism Design Approach. American Economic Review, 93: 729-747.

Agranov, Marina and Pietro Ortoleva. 2013. Stochastic Choice and Hedging. Mimeo.

Anderson, Christopher J. 2003. The Psychology of Doing Nothing: Forms of Decision Avoidance Result From Reason and Emotion. Psychological Bulletin, 129: 139-167.

Andreoni, James and Charles Sprenger. 2011. Uncertainty Equivalents: Testing the Limits of the Independence Axiom. National Bureau of Economic Research Working Paper 17342.

Bartling, Björn and Urs Fischbacher. 2012. Shifting the Blame: On Delegation and Responsibility. Review of Economic Studies, 79: 67-87.

Beattie, Jane, Baron, Jonathan, Hershey, John C. and Mark D. Spranca. 1994. Psychological determinants of decision attitude. Journal of Behavioral Decision Making, 7: 129-144.

Berg, Joyce E., Dickhaut, John W. and Thomas A. Rietz. 2010. Preference Reversals: The Impact of Truth-Revealing Monetary Incentives. Games and Economic Behavior, 68: 443-468.

Bolton, Gary E., Brandts, Jordi and Axel Ockenfels. 2005. Fair Procedures: Evidence from Games Involving Lotteries. Economic Journal, 115: 1054-1076.

Borah, Abhinash. 2010. Other-Regarding Preferences and Procedural Concerns. Mimeo.

Braun, Sebastian and Nadja Dwenger. 2009. Success in the University Admission Process in Germany: Regional Provenance Matters? Higher Education: The International Journal of Higher Education and Educational Planning, 58: 71-80.

Braun, Sebastian, Dwenger, Nadja, Kübler, Dorothea and Alexander Westkamp. 2012. Implementing Quotas in Matching Markets: An Experimental Analysis, Social Science Research Center Berlin (WZB), Discussion Paper No. SP II 2012-201.

Braun, Sebastian, Dwenger, Nadja, and Dorothea Kübler. 2010. Telling the Truth May Not Pay Off: An Empirical Study of Centralized University Admissions in Germany. The B.E. Journal of Economic Analysis 85 Policy (Advances) 10: Article 22.

Buchstein, Hubertus. 2009. Demokratie und Lotterie. Das Los als polititisches Entscheidungsinstrument von der Antike bis zur EU, Campus, Frankfurt/Main, New York.

Camerer, Colin F. and Teck-Hua Ho. 1994. Violations of the Betweenness Axiom and Nonlinearity in Probability. Journal of Risk and Uncertainty, 8: 167-196.

Chen, Yuh-Jia and James E. Corter. 2006. When Mixed Options are Preferred in MultipleTrial Decisions. Journal of Behavioral Decision Making, 19: 17-42.

Diecidue, Enrico, Schmidt, Ulrich and Peter P. Wakker. 2004. The Utility of Gambling Reconsidered. Journal of Risk and Uncertainty, 29, 241-259. 
Gale, David and Lloyd Shapley. 1962. College Admissions and the Stability of Marriage. American Mathematical Monthly, 69: 9-15.

Gilovich, Thomas and Victoria H. Medvec. 1995. The Experience of Regret: What, When, and Why. Psychological Review, 102: 379-395.

Gneezy, Uri, List, John A., and George Wu. 2006. The Uncertainty Effect: When a Risky Prospect is Valued Less than its Worst Possible Outcome. Quarterly Journal of Economics, 121: 1283-1309.

Haeringer, Guillaume and Flip Klijn. 2009. Constrained School Choice. Journal of Economic Theory, 144: 1921-1947.

Kahneman, Daniel, Knetsch, Jack L. and Richard Thaler. 1986. Fairness as a Constraint on Profit Seeking: Entitlements in the Market. American Economic Review, 76: 728-741.

Kahneman, Daniel, and Dalel T. Miller. 1986. Norm Theory: Comparing Reality to its Alternatives. Psychological Review, 93: 136-153.

Krawczyk, Michal and Fabrice Le Lec. 2010. Give Me a Chance! An Experiment in Social Decision Under Risk. Experimental Economics, 13: 500-511.

Keren, Gideon and Karl H. Teigen. 2010. Decisions by Coin Toss: Inappropriate But Fair. Judgement and Decision Making, 5: 83-101.

Leonhardt, James M., Keller, Robin L., and Cornelia Pechmann. 2011. Avoiding the Risk of Responsibility by Seeking Uncertainty: Responsibility Aversion and Preference for Indirect Agency When Choosing for Others. Journal of Consumer Psychology, 21: 405413.

Loomes, Graham and Robert Sudgen. 1982. Regret Theory: An Alternative Theory of Rational Choice Under Uncertainty. Economic Journal, 92: 805-824.

Machina, Mark. 1989. Dynamic Consistency and Non-Expected Utility Models of Choice Under Uncertainty. Journal of Economic Literature, 27: 1622-1668.

Roth, Alvin E. 1982. The Economics of Matching: Stability and Incentives. Mathematics of Operations Research, 7: 617-628.

Roth, Alvin E. 2008. Deferred Acceptance Algorithms: History, Theory, Practice, and Open Questions. International Journal of Game Theory, 36: 537-569.

Roth, Alvin E. and Elliott Peranson. 1999. The Redesign of the Matching Market for American Physicians: Some Engineering Aspects of Economic Design. American Economic Review, 89: $748-780$.

Roth, Alvin E. and Uriel G. Rothblum. 1999. Truncation Strategies in Matching Markets-in search of Advice for Participants. Econometrica, 67: 21-43.

Roth, Alvin E. and John H. Vande Vate. 1991. Incentives in Two-Sided Matching with Random Stable Mechanisms. Economic Theory, 1: 31-44. 
Rubinstein, Ariel. 2002. Irrational Diversification in Multiple Decision Problems. European Economic Review, 46: 1369-78.

Sen, Amartya. 1997. Maximimization and the Act of Choice. Econometrica, 65: 745-779.

Sonsino, Doron. 2008. Disappointment Aversion in Internet Bidding Decisions. Theory and Decision, 64: 363-393.

Wakker, Peter P. 2010. Prospect Theory: For Risk and Ambibuity, Cambridge University Press, Cambridge.

Westkamp, Alexander. 2013. An Analysis of the German University Admissions System. Economic Theory, 53: 561-589.

Zeelenberg, Marcel and Rik Pieters. 2007. A Theory of Regret Regulation 1.0. Journal of Consumer Psychology, 17(1): 3-18. 


\section{A Appendix}

\section{A1 Proofs}

Proof of Proposition 1: (i) Since neither $a_{1}$ nor $a_{2}$ are weakly optimal for all $s \in S$, it holds that $m\left(\mathcal{L}_{1,2}, \mathcal{A}\right) \subset m\left(a_{i}, \mathcal{A}\right)$ for both $i=1,2$. Therefore, responsibility loving requires that $a_{i} \succsim \mathcal{L}_{1,2}$ implies $a_{i} \succsim_{\mathcal{A}} \mathcal{L}_{1,2}$. From the fact that $\succsim$ satisfies betweenness (because it satisfies expected utility), $a_{i} \succsim \mathcal{L}_{1,2}$ must hold for at least one $i=1,2$.

(ii) Assume w.l.o.g. that $a_{1} \succsim a_{2}$. Since expected utility satisfies betweenness, it follows that $a_{1} \succsim \mathcal{L}_{1,2} \succsim a_{2}$. A responsibility averter dislikes the larger responsibility sets of $a_{1}$ and $a_{2}$ relative to $\mathcal{L}_{1,2}$ and can therefore violate betweenness: consider the preference order $\mathcal{L}_{1,2} \succ_{\mathcal{A}} a_{1} \succsim_{\mathcal{A}} a_{2}$. Using the observation from part (i) that $m\left(\mathcal{L}_{1,2}, \mathcal{A}\right) \subset m\left(a_{i}, \mathcal{A}\right)$ for both $i=1,2$, it is straightforward to check that this preference order satisfies Definition 2 (ii).

Proof of Proposition 2: Because of the strictness of preference $\succsim$, it must hold that $\mathcal{L}_{1,2} \succ$ $\mathcal{L}_{2,1}$. It also holds that $m\left(\mathcal{L}_{1,2}, \mathcal{A}\right)=m\left(\mathcal{L}_{2,1}, \mathcal{A}\right)$ : both lotteries render the same actions possible independent of the state $s$ and each lottery can therefore be suboptimal in state $s$ if and only if the other lottery can be suboptimal in $s$. The two observations together imply by definition that a responsibility averter with preference $\succsim_{\mathcal{A}}$ prefers $\mathcal{L}_{1,2}$ over $\mathcal{L}_{2,1}$.

\section{A2 The Boston algorithm used in procedure A}

The Boston algorithm applied in procedure A can be described as follows:

Step 1: Only the first preferences of the applicants are considered. For each university, admit the selected applicants who have ranked it as their first choice, until there are no seats left or until all candidates ranking this university as their first choice have been admitted. If there are more candidates giving priority to a university than can be admitted, those applicants with the best grades in the Abitur are admitted. Social criteria and (subordinately) lotteries are used to break ties.

Step $k$ : Only the $k^{t h}$ preference of the still unassigned applicants is considered. For each university with available seats, admit the selected applicants who have ranked it as their $k^{\text {th }}$ choice, until there are no seats left or until all candidates ranking the university as their $k^{\text {th }}$

choice have been admitted. If there are more candidates giving the rank $k$ to a university than can be admitted, those applicants with the best average final grade from school are admitted. Social criteria and (subordinately) lotteries are used to break ties.

The algorithm stops after step $k \leq 6$ when every selected applicant is assigned or when all 6 preferences have been considered.

\section{A3 The Gale-Shapley algorithm used in procedure U}

The Gale-Shapley algorithm applied in procedure U works as follows:

Step 1: Each university $i$ with capacity $n_{i}$ offers a seat to the $n_{i}$ applicants it ranks highest. Each applicant tentatively accepts the offer from the university she ranks highest and rejects all remaining offers. 
Step $k$ : Each university that was rejected at step $k-1$ by $x$ applicants proposes to its most preferred next choices, with the number of new offers $(x)$ being equal to the number of rejections in the previous round. Each applicant considers the university it has been holding an offer from together with her new offers and tentatively accepts the university she ranks highest and rejects all others.

The algorithm terminates when no proposal by a university is rejected. Each university and applicant is assigned according to the last tentative assignment. If for a certain seat a university is rejected by all applicants to which it has made an offer and there is no applicant left on its preference list, this seat remains unfilled.

\section{A4 Experiment II: Instructions and decisions sheets-FOR ONLINE PUB- LICATION}

\section{A4.1 Instructions}

\section{Instructions}

In the following part of today's lecture you will participate in a decision experiment for which we have just distributed a decision sheet. We ask you to please carefully read this instruction sheet first and then the decision sheet. With a bit of luck you can receive monetary prizes, depending on your entries on the decision sheet. These prizes will be described further below and they will be distributed at the end of this lecture.

Your entries will serve as data for current research projects by the TU Berlin, the HU Berlin, the WZB (Social Science Research Center Berlin), the DIW (German Institute for Economic Research) and the Max Planck Institute for Tax Law and Public Finance (Munich). Your data will remain entirely anonymous.

It is crucial for the success of this experiment that you make your entries independently of each other. From this moment on do not speak to the other participants until the end of the experiment and do not look at their decision sheets. Also, please do not make any loud noises or create other disruptions. If you do not abide by these instructions, we will exclude you from the possibility of receiving a prize. Thank you.

If you have any questions, please alert the experiment conductor via a hand signal. We will then approach you in order to answer your question discreetly and quietly.

\section{Random determination of the winners}

Five out of 100 experiment participants will be randomly selected and will receive their prizes according to their decision sheets. To this purpose we will randomly select code numbers with a computer subsequent to the experiment, that is, during the proceeding lecture. These draws will determine who is entitled to receive a prize. Therefore, it is important that you keep the piece of paper with the code number and that you write down your code number at the top of your decision sheet. The computer will select a certain amount of numbers so as to exactly enable one out of 20 participants in each of the (started) groups to receive their payout. For instance, with 300 participants 15 code numbers will be randomly chosen. With a number of participants between 301 and 320, 16 code numbers will be randomly chosen, and so forth. 


\section{Possible prizes}

You can win the following vouchers - depending on your choices on the following page and the rules given there. The vouchers can be used immediately and are valid for all products that can be purchased at all Starbucks stores or at amazon.de respectively (with the exception of magazines and download products).

Alternative A: Starbucks voucher over the amount of 19 Euro

Alternative B: Amazon voucher over the amount of 19 Euro

\section{A4.2 Decision sheet, binary-choice format}

We would now like to ask you to choose two times between the Alternatives A and B, in "Option (i)" and "Option (ii)".

Only one of the two choices will be relevant for the determination of the prize, though Option (i) will be more probable than Option (ii) with a ratio of 60/40. To this purpose we will later simulate a random draw on the computer, which will result in a randomly chosen number between 1 and 100, where every integer in this interval occurs with the same probability. If the random draw results in a number between 1 and 60, Option (i) is applied. Your payoff, and the payoffs of all other participants in the experiment, will only be determined by Option (i). Thus, this will occur with a probability of $60 \%$. If, however, the random draw results in a number between 61 and 100 (which will occur with a probability of 40\%) only Option (ii) will be implemented. You can decide whether to choose different alternatives for both options or if you would prefer to choose the same alternative twice.

Please state both your selection choices below.

Option (i): Which one of the Alternatives A/B would you prefer to receive?

Option (ii): Which one of the Alternatives A/B would you prefer to receive?

Please do not forget to write down your code number at the top. As soon as all participants have filled in their choices, we will collect the decision sheets and we will determine the winners as well as the prizes during the proceeding lecture. At the end of the lecture we will then announce which code numbers won. Please remain silent. In case of questions questions, please raise your hand.

\section{A4.3 Decision sheet, four-way-choice format}

We would now like to ask you to choose from the following 4 possible options (Option 1 to Option 4), which in some cases result in a random selection of prizes.

Option 1:

You will receive Alternative A with a probability of $60 \%$ and Alternative B with a probability of $40 \%$. 
Option 2:

You will receive Alternative A with a probability of $100 \%$.

Option 3:

You will receive Alternative B with a probability of $60 \%$ and Alternative A with a probability of $40 \%$.

Option 4:

You will receive Alternative B with a probability of $100 \%$.

As you can see, the random draw which determines the prize for Options 1 and 3 follows two probabilities with a ratio of respectively $60 / 40$. To this purpose we will later simulate a random draw with the computer, which will result in a randomly drawn number between 1 and 100 , where each integer in this interval occurs with the same probability. If the random draw results in a number between 1 and 60, the primarily stated alternative will be implemented for you as well as for all other experiment participants who have chosen Option 1 or Option 3. Thus, this will occur with a probability of $60 \%$. If, however, the random draw results in a number between 61 and 100 (which will occur with a probability of 40\%) the alternative that was named in the second place will be implemented. If you choose Option 2 or Option 4 , which can only result in one alternative, this random draw is irrelevant for you.

Please state your choice here:

Which one of the four Options would you like to choose?

Please do not forget to write down your code number at the top. As soon as all participants have filled in their choices, we will collect the decision sheets and we will determine the winners as well as the prizes during the proceeding lecture. At the end of the lecture we will then announce which code numbers won. Please remain silent. In case of questions questions, please raise your hand. 